\title{
El concepto de potencia en las relaciones internacionales
}

\section{Luis V. Pérez Gil}

El autor reflexiona acerca de la vigencia del concepto de potencia, partiendo de la base que una potencia es más que un Estado-nación. Define a las potencia como aquellos Estados que establecen las reglas del sistema y que disponen de los recursos y de las capacidades necesarias para movilizarlos de forma optima en defensa de dichas reglas. Aunque la dimensión fisica en muy importante, no es el único elemento para valorar la capacidad de un Estado en el sistema intemacional. El poder militar también ha sido siempre uno de los primeros atributos a tener en cuenta para la valoración de un Estado, pero además, debe contar con excelentes recursos materiales, industriales y militares, sustentados en una base económica floreciente. Finaimente, señala que el poder es requisito fundamental, pere es imprescindible la voluntad del Estado de usar dicho poder.

\section{Introducción}

El 11 de septiembre de 1990 el presidente Bush anunció, en sesión conjunta del Congreso y Senado de los Estados Unidos, el objetivo del establecimiento de un nuevo orden internacional, de una "nueva era, más libre de la amenaza del terror, más vigorosa en la realización de la justicia y más segura en la búsqueda de la paz, una era en la que las naciones de todo el mundo, Este y Oeste, Norte y Sur, puedan prosperar y vivir en armonía." Pareció entonces que asistíamos a la inauguración de una nueva etapa en la conducción de los asuntos internacionales, "donde la ley del Derecho sustituye a la ley de la selva, un mundo donde las naciones reconocen la responsabilidad compartida por la libertad y la justicia, un mundo donde el fuerte respeta los derechos del débil" . Sin embargo, para disgusto de la pléyade de ingenuos y utópicos 
sin remisión que pueblan en el mundo de las relaciones internacionales, se trataba simplemente de la renovación de la promesa americana -y van tres en este siglo- de iluminar al mundo con las luces de la virtud otorgada por el "destino manifiesto" que, Administración republicana o demócrata vigente poco importa, retoza cada mañana sobre el lecho mesiánico proclamado por el "visionario" presidente Wilson en 1916: "Fue como si en la providencia de Dios un continente se hubiese mantenido virgen aguardando a un pueblo pacífico que amara la libertad y los derechos más que ninguna otra cosa, para que llegara a establecer una comunidad de auténtico desinterés" 2 .

De ambas proclamas presidenciales, separadas casi un siglo en el tiempo, destacamos dos ideas fundamentales: 1) el deseo de los Estados Unidos de contribuir al establecimiento de un orden internacional más justo e igualitario para todos; y 2) la persistente apelación, como norma básica de ese orden internacional, al imperio de la ley, donde el fuerte respeta los derechos del débil. Sendos postulados se deben desarrollar, obviamente, en el marco de una indiscutible supremacía americana ${ }^{3}$. En 1919 esta supremacía era de orden eminentemente ideológico, convirtiéndose el presidente Wilson en el gran impulsor de la primera organización internacional de carácter general, la Sociedad de Naciones, con la finalidad primordial de velar por la paz y la seguridad interṇacionales ${ }^{4}$. En la última década de este siglo, engullida la Unión

\footnotetext{
' Bush: Towards a New World Order. U.S. Department of State. Washington, 1990 (cit. en Remiro Brotons: "Civilizados, bárbaros y salvajes en el nuevo orden internacional", Cursos de Derecho Internacional de Vitoria Gasteiz, 1994, pp. 17-84, en concreto, p. 21, la cursiva es nuestra).

2 Discurso del presidente Wilson el día de la graduación de la Academia Militar de West Point, 13 de junio de 1916 (cit. en Kissinger: Diplomacia. Ediciones B. Barcelona, 1996 (trad. de Diplomacy. S.e., 1994), p. 42, la cursiva es nuestra). El presidente Bush nos recordaba hace poco estas palabras (1993): "Vivimos en un país resplandeciente en libertad, donde la gente de todas las religiones, razas, credos y colores pueden esperar vivir en paz y armonía; donde los éxitos y los logros son determinados por la iniciativa individual y la imaginación; donde hay una creencia ilimitada en la dignidad humana y en el potencial humano; donde siempre existe la esperanza de un mañana mejor. Nosotros debemos continuar compartiendo 'nuestras esperanzas y nuestros sueños con el mundo." (National Security Strategy of the United States. The White House. Washington, 1993, p. 21, la cursiva es nuestra). En idéntico sentido se expresó el presidente Clinton en su primer discurso sobre el "estado de la Unión" en enero de 1994 (en New York Times, 26 de enero de 1994), y más reciente, en A National Security Strategy for a New Century. The White House. Washington, 1997.

${ }^{3}$ Uno de los más autorizados panegíricos de la nueva era lo realiza Krauthammer en "The unipolar moment", For. Aff. núm. 70, 1991, pp. 23-33. Este autor propone un mundo unipolar bajo el liderazgo de los Estados Unidos como poder militar dominante entregado a la misión de instaurar la democracia, de ser necesario por la fuerza y sin respetar las normas básicas del Derecho internacional (el principio de la igualdad soberana de los Estados, la no injerencia en los asuntos internos, ...).

${ }^{4}$ Sobre el particular, vid. Pêrez Gil: "El primer decenio de España en la Sociedad de Naciones (19191929)", Anales de la Facultad de Derecho de la Universidad de La Laguna núm. 15, 1998, pp. 175-215, en concreto pp. 176*189.
} 
Soviética por la ley inexorable de la historia -los imperios nacen y mueren-, los actores del sistema internacional han sido testigos del despliegue masivo de poder de que hacen gala los Estados Unidos, sin parangón en la historia de las relaciones internacionales. Y como afirmó Morgenthau, "lo que importa en una sociedad de naciones soberanas es dónde reside el mayor poder" 5 .

Con el trasfondo del definitivo encumbramiento de la primera potencia hegemónica del sistema internacional global, nos proponemos reflexionar, aun sea brevemente, sobre la vigencia de uno de los temas centrales en el análisis de las relaciones internacionales: el concepto de "potencia". En efecto, como trataremos a continuación, todos los autores que se han acercado al estudio de los asuntos internacionales y de la política exterior de los Estados realistas y científicos por igual- no han podido resistirse a la tentación de apelar a la noción de potencia e, inmediatamente, proceder a calificarla estableciendo un orden jerárquico entre ellas. Es más, las clasificaciones de los sistemas internacionales que han propuesto los teóricos de las relaciones internacionales se estructuran en torno a la noción de "potencia".

\section{Noción de "potencia" y sistema internacional}

La primera y más simple acepción que nos encontramos del término "potencia" en relaciones internacionales es la de "Estado o Nación soberana" -acepción que recoge también el Diccionario de la Lengua Española ${ }^{6}$ El uso del término "potencia" con este significado se desarrolló ampliamente en el concierto diplomático del siglo XIX para referirse a todas las naciones que participaban en el sistema europeo de Estados ${ }^{7}$. No obstante, para nuestro objeto de estudio, esta acepción de "potencia" no interesa. Cuando aludimos al término "potencia" nos estamos refiriendo a algo más que un "Estado o Nación" ".

\footnotetext{
5 La lucha por el podery por la paz. Ed. Sudamericana. Buenos Aires, 1963 (trad. de Politics amog Nations. The Struggle for power and peace. A Knopf. Nueva Yok, 1960), p. 588.

6 Diccionario de la Lengua Española. Real Academia Española (21 ${ }^{\mathrm{a}}$ ed.). Madrid, 1992.

7 El concierto europeo de potencias era un sistema diplomático en el cual concumín las principales potencias europeas para la resolución de sus controversias. No era un sistema ńgido ni basado en pactos que pretendieran colocar a todos los países en pie de igualdad. Se reunía cuando lo requerían las circunstancias, y los Estados que lo formaban conservaban intacta su soberanía. Fracasó al no evitar la guema entre los Estados europeos -Crimea en 1854 y franco-prusiana en 1870-71-; sin embargo, evitó la formación de bloques permanentemente hostiles entre grupos de Estados hasta finales del siglo XIX. En este punto, vid, in extenso Kissinger: Diplomacia, op. cit., pp. 77-171.
} 
Resulta evidente en la realidad de los asuntos internacionales que la diferencia de tamaño tiene consecuencias políticas amplísimas, tanto para los grandes Estados como para las naciones pequeñas. El Estado con mayores recursos económicos y mayor población tiene más influencia fuera de sus fronteras, mayor seguridad frente a las presiones, las amenazas y el ataque militar y, en definitiva, más prestigio y más amplio campo de elección en la elaboración y ejecución de su política exterior. Por el contrario, un Estado pequeño es más vulnerable, menos resistente a las presiones del exterior, más limitado en sus opciones políticas y mantiene una vinculación -por limitaciónmás estrecha entre su política interior y su política exterior ${ }^{9}$. En consecuencia, cuanto más pequeño es un Estado menos viable será como miembro independiente de la sociedad internacional desde un punto de vista material puesto que, desde un punto de vista formal, el principio de igualdad soberana entre los Estados se halla en la base del ordenamiento jurídico internacional y ha sido consagrado en todos los textos jurídicos internacionales de este siglo ${ }^{10}$. Parece, pues, una afirmación casi obvia que los Estados iguales en derecho son desiguales de hecho ${ }^{11}$, y por tanto, para acceder al concepto de potencia cuentan sobremanera los elementos material y espiritual del Estado -ya vere-

\footnotetext{
${ }^{8}$ En este sentido, Rubio García: "Significado de los Estados pequeños y medios", Rev. Pol. Int. núm. 104, 1969, pp. 55-65, en concreto pp. 62.

9 Vital: La desigualdad de los Estados. Estudio de las pequeñas potencias en las relaciones internaciona. les. Instituto de Estudios Políticos. Madrid, 1976 (trad. de The Inequality of States. A Study of Small Power in International Relations. Clarendion Press. Oxford, 1967), p. 19; Reynolds: Introducción al estudio de las relaciones internacionales. Tecnos. Madrid, 1977 (trad. de An Introduction to interrational relations. Logman Group Ltd. Londres, 1971), p. 160.

${ }^{10}$ El artículo 10 del Pacto de la SdN estableció que "Los Miembros de la Sociedad se comprometen a respetar y a mantener contra toda agresión exterior la integridad territorial y la independencia política presente de todos los miembros de la Sociedad". Más tarde, la idea de la igualdad soberana de los Estados se recogió en el artículo 2.1 de la Carta de la ONU expresando claramente que "La Organización está basada en el principio de la igualdad soberana de todos sus Miembros". A pesar de estas declaraciones, Oppenheim puso de manifiesto que el reconocimiento legal de las grandes potencias encontró una base jurídica con el establecimiento del Consejo de la $5 \mathrm{dN}$, circunstancia confirmada más tarde con la creaciôn del Consejo de Seguridad por la Carta de la ONU. Sobre esta cuestiôn, para consultar un clásico, vid. Oppenheim: Tratado de Derecho Internacional Público (4 vols.). Ed. Bosch. Barcelona, 1961 (trad. de International Law. A treatise (Lauterpacht ed.). Logman, Green and Co ( $8 \mathrm{a}$ ed.). Londres, s.d.), vol. $1-1, \mathrm{pp}$. 278-300; para una perspectiva actual, Pastor Ridruejo: Curso de Derecho Internacional Püblico y Organizaciones Internacionales. Tenos ( $5 \stackrel{a}{\text { ed.). }}$. Madrid, 1994, pp. 293-301. En concreto sobre dicho principio en la Carta de la ONU, vid. Mbaye: "Article 2 Paragraphe 1", en La Charte des Nations Unies (Cot y Pellet dir.). Economica. París, 1985, pp. 79.96.

"Merle: Sociología de läs relaciones internacionales. Alianza Editorial ( $2^{ \pm}$ed. ampl.). Madrid, 1991 (trad. de Sociologie des relations internationales. Jurisprudence Génerale Dalloz (4 ed.). País, 1988), p. 346. En este sentido, el juez Anzilotti, en su opinión particular al dictamen del TPJl de 5 de septiembre de 1931 sobre la Unión Aduanẹra Austro•alemana, expresố claramente que "la concepciốn juridica de la independencia no tiene nada que ver con los estados de dependencia de facto cada vez más numerosos que caracterizan la relación de un país con otro." (CPJI série A/B Part l, pp. 57•58).
} 
mos más adelante en qué extensión-. Así, el reconocimiento jurídico de la condición de potencia, especialmente de las "grandes potencias", no es sino la mera constatación de una situación preexistente. Esto es así por cuanto que, como ya afirmó Schwarzenberger, en esta etapa de desarrollo de la sociedad intemacional, "Ios Estados apelan a la aplastante ley de la fuerza y la supremacía del poder se reafirma" ${ }^{12}$.

De esta manera, podemos decir que existen al menos tres tipos de Estados que deben tomarse en consideración en el análisis del concepto de potencia ${ }^{13}$. El primero de estos tipos es el Estado cuyo interés principal reside en sí mismo y en los países vecinos y que, en palabras de Merle, ejerce una función local. La fuerza que estas naciones puedan desarrollar se ve limitada a una esfera geográfica muy localizada; ni que decir tiene que la mayoría de los Estados del sistema internacional pertenecen a esta primera categoría, equivalente a la acepción de "potencia" desechada anteriormente. El segundo tipo lo constituyen aquellos Estados con influencia en un sector particular de las relaciones internacionales y/o con responsabilidades regionales, que han sido tradicionalmente denominados "potencias medias" o "potencias regionales". Estos Estados no son potencias mundiales porque carecen de los recursos necesarios para ostentar tal rango, pero sus responsabilidades no están limitadas a los países vecinos de su entorno. El tercer tipo de Estados lo forman aqueIlos cuyos recursos, intereses y capacidades militares son tales que pueden hacer sentir su influencia en todos los asuntos mundiales y alcanzar sus objetivos de una forma más plena que los otros miembros de la sociedad internacional; se trata de las "grandes potencias" 14. Como ha destacado Merle, "la

\footnotetext{
12 La política del poder. Fondo de Cultura Económica. México, 1960 (trad. de Power Politics. A study of international society. Stevens and Sons Ltd. Londres, 1951), p. 97; Morgenthau: La lucha por el poder y por la paz, op. cit., p. 588; Deutsch: Análisis de las relaciones internacionales. Ed. Gernika ( $3^{\mathrm{a}}$ ed.). México, 1994 (trad. de Analysis of International Relations. Prentice-Hall. Englewood (N.J.), 1988), p. 209. ${ }^{23}$ Merle hace una clasificación cuatripartita de los Estados de acuerdo con la capacidad de los actores estatales para asumir una "función" más o menos amplia en las relaciones internacionales (Sociologia de las relaciones internacionales, op. cit., pp. 348.355). Sustancialmente, estamos de acuerdo con esta clasificación aunque, posiblemente y como hemos hecho, pueda ser reducida a tres tipos de Estados de acuerdo con el papel que son capaces de desemperiar en los asuntos internacionales.

4 El sistema europeo de Estados siempre ha estado dominado por unas cuantas potencias. Del caos de la Guerra de los Treinta Años surgió una Europa cuyo orden político fue organizado por un grupo selecto de los más poderosos Estados, que se reconocían mutuamente como las "Grandes Potencias". Más tarde, en el concierto europeo del siglo XIX vimos como se generalizó el uso del têrmino para referirse a los componentes del directorio del sistema europeo de Estados. Ya en 1919, en la Conferencia de Paz de Versalles, la designaciōn de "grandes potencias" fue atribuida a cinco delegaciones, y la de "potencias menores" a tres. La SdN y la ONU han perpetuado, y modificado en parte, esta tradición que fue formalmente iniciada en el período del Congreso de Viena. Como expresan Russett y Starr, se trata simplemente de reconocer la realidad del poder (World Politics. The Menu for Choice. Freeman and Co. (5 ${ }^{\underline{a}}$ ed.) Nueva York, 1996, p. 60).
} 
combinación de estos factores (que analizaremos a continuación) constituye la base del poderío material que ofrece a estos Estados la posibilidad de intervenir (diplomática y) militarmente en todos los puntos del globo donde sus intereses están en juego" 15 .

A.sí pues, cuando nos referimos a las "potencias" hablamos de Estados, pero de Estados con ciertas particularidades de tipo material y subjetivo - en este punto, que para nosotros es el elemento decisivo, insistiremos más adelante-, que hacen que sobresalgan por encima de sus iguales. Es decir, hablamos de Estados capaces de mantenerse firmes ante cualquier otra nación, y por eso se elevan a la categoría de sujetos activos en las relaciones internacionales.

Algunos autores responden a la pregunta de qué es una potencia atendiendo a sus recursos y a la capacidad de movilización de los mismos. Otros tienen en consideración el efecto que causaría en el sistema la aparición de una potencia -sea mundial o regional, grande o media-. Por tanto, podemos decir que potencias son aquellos Estados que establecen las reglas del sistema y que disponen de los recursos y de las capacidades necesarias para movilizarlos de forma óptima en defensa de dichas reglas ${ }^{16}$. Así, los actoresplayers en la terminología americana- que configuran la estructura del sistema son indudablemente las potencias, pues, como ha dicho Barbé, "sólo ellas disponen del poder estructural que permite dictar las reglas de juego a nivel global en el sistema internacional" 17; el resto de los Estados del sistema "son mucho más objetos que sujetos de las relaciones internacionales"18.

Tanto es así que el número de potencias ha sido el criterio adoptado tradicionalmente para determinar la tipología de los sistemas internacionales o regionales de Estados: unipolar o imperial, bipolar y multipolar ${ }^{19}$. El establecimiento de una tipología cuantitativa de los sistemas internacionales no es caprichosa y así, autores como Aron, Bull, Hoffmann, Holbraad, Kaplan o Waltz, acuden también a este criterio para fijar una clasificación de los siste-

\footnotetext{
is Sociologia de las relaciones internacionales, op. cit., p. 351.

${ }^{16}$ En esta línea, Modelski define a las potencias mundiales como aquellos Estados que se encargan, en mayor medida que los demás, del "mantenimiento del orden" en el sistema político global (cit. por Taylor: Geografía política. Trama Editorial. Madrid, 1994 (trad. de Political Geography: World Economy, NationState and Locality. Logman Group (3 ${ }^{\mathrm{a}}$ ed.). Londres, 1993), p. 60).

$"$ "La estructura del sistema internacional ha sido definida por Barbé "como la configuración de poder surgida de las relaciones entre actores" (Relaciones Internacionales. Tecnos. Madrid, 1995, p. 197-198).

${ }^{28}$ Merle: Sociología de las relaciones internacionales, op. cit., p. 355.

"Holbraad establece una terminología diferente: sistema unifocal, dualista, triangular y múltiple (Las potencias medias en la política internacional. Fondo de Cultura Económica. México, 1989; trad. de Middle Powers in International Politics. The MacMillan Press. Londres, 1984).
} 
mas internacionales. Otros recurren a un segundo criterio para el análisis del fenómeno. Se trata de la referencia a los sistemas de valores de los Estados que integran el sistema internacional. Entre ellos, Aron habla de sistemas homogéneos y sistemas heterogéneos atendiendo a sí quienes los integran pertenecen al mismo tipoy obedecen almismo concepto de política ${ }^{20}$. Así la definición de "potencia" tiene en cuenta el volumen de los recursos y el sistema en el que se inserta.

\section{Los atributos: recursos económicos y militares}

Si la historia del sistema de Estados europeo, y más tarde internacional, se caracteriza por el auge y caída de determinadas potencias principales, ${ }^{21} \mathrm{en-}$ tonces en el análisis del fenómeno surge una pregunta inevitable: ¿cómo ciertos Estados escalan a la condición de potencias, e incluso pueden llegar a convertirse en potencias dominantes -" superpotencias ", en la terminología de la guerra fría ${ }^{22}$-, dando carta de naturaleza a uno u otro sistema? La respuesta no es sencilla puesto que el fenómeno en sí tampoco lo es ${ }^{23}$.

La dimensión física del Estado, aunque de gran importancia desde el punto de vista de los recursos naturales y capacidades económico-productivas, no representa el único elemento para valorar su capacidad en el sistema internacional. ${ }^{24}$ Efectivamente, el nivel de desarrollo económico, la situación geográfica y la naturaleza del medio en el que el Estado está situado, la cohesión de su elemento subjetivo, la fortaleza del gobierno y, finalmente, la influencia alcanzada en el sistema internacional son importantes factores que mo-

\footnotetext{
${ }^{20}$ Aron: Paz y guerra entre las naciones (2 vols.). Alianza Editorial. Madrid, 1985 (trad. de Paix et guerre entre les nations. Calmann-Lévy. París, 1984), vol. 1, p. 140-145.

"2 Schwarzenberger: La política del poder, op. cit., pp. 89-91; Taylor: Geogralia política, op. cit., p. 59; y en general Kennedy: Auge y caída de las grandes potencias. Plaza y Janés. Barcelona, 1994 (trad. de Rise and Fall of the Great Powers. S.e. 1987).

${ }^{22}$ El primer autor que utilizó el têrmino para referirse a las grandes potencias mundiales que surgirían de la Segunda Guerra Mundial fue Fox: Las superpotencias. Fondo de Cultura Económica. México, 1944 (trad. de The Superpowers. Yale Institute of International Studies, 1944).

${ }^{23}$ En este punto es interesante recordar las palabras de Ledermann: "La historia, esa gran consoladora, está ahí, una vez más, para hacernos ver que sobre el plan internacional, la potencia política, militar y económica es efímera. [...] Ayer una gran potencia, hoy un pobre Estado, podría decirse". (Considerations sur le Petit État. Neuchâtel, 1946, pp. 62-63).

${ }^{24}$ Sobre la influencia del medio fisico en el comportamiento de los Estados, vid. Morgenthau: La lucha por el poder y por la paz, op. cit., pp. 151-160; Renouvin y Duroselle: Introducción a la política internacional. Ed. Rialp. Madrid, 1968 (trad. de Introduction à l"histoire des relations internationales. Armand Colin. Paris, s.d.), pp. 27-35; Reynolds: Introducción al estudio de las relaciones internacionales, op. cit., pp. 6676; y en general Taylor: Geografía política, op. cit.
} 
difican la capacidad de un Estado -su base de poder-para actuar como sujeto activo en la sociedad internacional . ${ }^{25}$ De todos modos, debemos destacar dos cuestiones. Primero, no hay duda de que la dimensión material fija unos límites a la acción exterior del Estado que difícilmente pueden ser superados, salvo, claro está, que se produzca la ampliación de sus límites materiales y poblacionales. $Y$, en segundo lugar, el poderío de un Estado también se encuentra afectado por las distintas formas en que sus gobernantes conciben e instrumentalizan las capacidades materiales del Estado. ${ }^{26}$

Tradicionalmente se ha considerado que el status de potencia se gana, o se pierde, tras el final de una guerra; ${ }^{27}$ pero esta idea es simplista. De todas maneras, la guerra es la forma extrema que tienen los Estados de demostrar el poder, como lo atestiguan las 224 guerras, tanto internacionales como conflictos de carácter interno que se produjeron entre 1816 y 1980 y que aparecen en la lista confeccionada por Small y Singer ${ }^{28}$ Un cúmulo de circunstancias en las que, sin duda alguna, pero no exclusivamente, el conflicto bélico juega un papel relevante, llevan a la aparición de una o varias potencias y al descenso de otras a la condición de meros sujetos pasivos, carentes de poder para modificar la estructura internacional. ${ }^{29}$

${ }^{25}$ Schwarzenberger: La politica del poder, op. cit., pp. 102-103; in extenso Morgenthau: La lucha por el poder y la paz, op. cit., pp. 151-203 y Renouvin y Duroselle: Introducción a la política internacional, op. cit., pp. 9-320. ${ }^{26}$ Reynolds: Introducción al estudio de las relaciones internacionales, op. cit., pp. 69-70; Vital: La desigualdad de los Estados, op. cit.i p. 20.

${ }^{27}$ Piénsese en los ejemplos de 1815,1919 y 1945. Como ha dicho Kennedy, la mayor parte de los autores, especialmente los geopolíticos, presumía que la guerra y el sistema de las grandes potencias iban de la mano. En este sentido, Mackinder sostenía que "las grandes guerras de la Historia [...] son resultado, directo o indirecto, del crecimiento desigual de las naciones". (cit. en Kennedy: Auge y caída de las grandes potencias, op. cit., p. 834). Acerca del pensamiento clásico de la geopolítica (Ratzel, Mackinder, Mahan, Haushofer), sometido a dura crítica tras la Segunda Guerra Mundial, vid. Gallois; Geopolítica. Los caminos del poder. Ediciones Ejército. Madrid, 1992 (trad. de Geopolitique. Les voies de la puissance. Fundation des Études de Défense Nationales.PLON. París, s.d.), pp. 225-364. Sobre este pensamiento clásico, sin duda impregnado de prejuicios nacionales, Taylor ha escrito recientemente: "tenemos que reconciliarnos con Mackinder y la geopolítica, no ignorarlos sino entender sus ideas en su contexto nacional e histórico. Sólo de esta manera podremos superar esta herencia, aunque deshonrosa, distinguida [sic]" (Geografía política, op. cit., p. 58). Para una reseña histórica sobre la nueva ciencia en España, vid. el excelente artículo de Truyol y Serra: "Sobre la introducción de la geopolítica en España", Hacia un nuevo orden internacional y europeo. Homenaje al profesor M. Díez de Velasco. Tecnos. Madrid, 1993, pp. 771-786. Desde el análisis sistémico, Ferris puso de manifiesto dos cuestiones importantes: 1) existe alguna relación entre el cambio en la distribución del poder en el sistema y la cantidad de guerras dentro del mismo; y, 2) cuanto mayor sea la disparidad de poder entre los Estados tanto más aumentará la posibilidad de que se involucren en conflictos intensos (The Power Capabilities of Nation-States. D.C. Heath. Lexington (Mass.), 1973, pp. 125-116).

${ }^{28}$ Resort to Arms: International and Civil Wars 18:6-1980. Sage. Beverly Hills, 1982. Exclusivamente con referencia a los conflictos internacionales, Holsti: Peace and War: armed conflict and international order 1648-1989. Cambridge University Press. Cambridge, 1991.

${ }^{2}$ Como expondremos măs adelante, el agotamiento es un factor de indudable importancia; agotamiento que no sólo es material, sino paralelamente de tipo moral y psicológico de las élites dirigentes del Estado. 
El poder militar de un Estado ha sido siempre el primer atributo a tener en cuenta para su valoración. ${ }^{30}$ Desde la antigüedad, las comunidades políticas en general, y más tarde los Estados, comprendieron que su prosperidad e influencia estaba relacionada directamente con su poderío militar. ${ }^{31}$ Teniendo en consideración la existencia de ejércitos permanentes, se podía confeccionar un cuadro de Estados clasificados por orden de sus fuerzas militares. ${ }^{32}$ Pero esta clasificación desviaba la atención de los hechos que determinan si esa fuerza militar es una amenaza para otro Estado o coalición de Estados. No obstante, es indudable que en términos generales existe una relación directa entre fuerza militar y poder político -influencia si se quiere-. Sin llegar al extremo de considerar a las armas como la ultima ratio regnum, según rezaba la leyenda inscrita en los cañones de los ejércitos de Luis XTV, resulta claro que el Estado que posee mayor poder militar tiende a ser el más influyente, sus deseos son los más respetados y su diplomacia la más efectiva; o como expresó Spykman "el poder es en última instancia capacidad de sostener una guerra." 33

${ }^{30}$ Como afirma Barbé "el tamaño militar del Estado constituye un criterio clásico a la hora de establecer jerarquías en el sistema internacional." (Relaciones internacionales, op. cit., p. 138); Keohane y Nye que "la fuerza militar es siempre un componente central del poder nacional" ("Realism and complex interdependence", Power and interdependence. World Politics in Transition. Little Brown. Boston, 1977, pp. 23-37); Morgenthau: "en política internacional, la fuerza armada como amenaza o como amenaza potencial, es el más importante factor que forma el poder político de una nación". Este autor disecciona el poder militar del Estado en: capacidad tecnológica, liderazgo militar y cantidad y calidad de las Fuerzas Armadas (La lucha por el poder y por la paz, op. cit., pp. 163-167). Con una perspectiva reciente, todo en general Brzezinski: El gran tablero mundial. La supremacia estadounidense y sus imperativos geopolíticos. Ed. Paidós. Barcelona, 1998 (trad. de The Grand Cheessboard. American Primacy and its Geostrategic Imperatives. BasicBooks, 1997).

${ }^{31}$ Esparta y Atenas, Roma y Cartago no son sino ejemplos clásicos de rivalidades que se resolvieron finalmente por la vía del conflicto bélico, poniendo en liza todo el poderío militar de que disponian ambos contendientes. Realmente, lo que estaba en juego era la prosperidad e influencia de ambos, en un caso en Grecia y en el otro en el Mare Nostrum. Sobre la permanencia del poder militar del Estado, Garnett realiza la siguiente reflexión: "Si el poder militar es electoralmente impopular y hay una dificultad inheren. te para su control, uno está tentado a preguntarse por qué los gobiernos no proceden a su abolición. Y la respuesta es que las serias preocupaciones por la adquisiciôn de fuerza militar son empequeñecidas por las preocupaciones de intentar arreglárselas sin ella. Dado el tipo de mundo en el que vivimos, el poder militar es visto por la mayoría de los estadistas como un prerrequisito de la supervivencia nacional." ("The role of military power", Perspectives on World Politics (Little y Smith ed.). Routledge ( $2^{\mathrm{a}}$ ed.). Londres, 1991, pp. 69-83, en concreto p. 71, la cursiva es nuestra). En este sentido, parece que Stalin preguntó una vez irónicamente "icuántas divisiones tiene?" refiriéndose al Papa, sin darse cuenta de que la reputación del Papado supera en mucho y es más perdurable en el tiempo que la de la mayoría de gobernantes seculares.

${ }^{32}$ Rubio García: "Significado de los Estados pequeños y medianos", op. cit., p. 56; Barbë habla incluso de "Estados armados", noción que nos parece excesiva a pesar de los argumentos que aporta en este sentido (Relaciones internacionales, op. cit., pp. 138-142).

33 Spykman: Estados Unidos frente al mundo. Fondo de Cultura Económica. México, 1944 (trad. de America"s Strategy in World Politics. Harcourt, Brace and Co., 1942), p. 47; Schwarzenberger: La política del poder, op. cit., pp. 103-104. 
A finales de los ochenta, sobre todo tras la caída del Muro de Berlín (1989) y la inauguración del "nuevo orden mundial" del Presidente Bush, muchos teóricos y estadistas -los ingenuos y utópicos sin remisión a los que nos referíamos al principio- comenzaron a hablar del declive de la fuerza militar como elemento de influencia en las relaciones internacionales, proceso similar al ocurrido en la etapa de deténte de los años setenta. Este estado de ánimo produjo, en palabras de Martin, "un difuso sentimiento de gran seguridad" en el que la fuerza militar parecía menos necesaria y, en definitiva, menos útil. ${ }^{34}$ Desgraciadamente, sólo un año después, la parafernalia del despliegue de la Guerra del Golfo -tanto material como sobre todo ideológica, de movilización de las masas de la gran potencia hegemónica- despertó de manera violenta a quienes pensaban que el poderío militar había sido desterrado como factor para valorar la influencia general de un Estado en la sociedad internacional y, en definitiva, para su consideración como potencia. ${ }^{35}$ Como ha dicho Howard "no es fácil imaginar cómo podrían ser conducidas las relaciones internacionales y mantenido el orden internacional, si [el poder militar] estuviese totalmente ausente."36 Por otro lado, no debemos olvidar que el poderío militar de las naciones cambió bruscamente a partir de 1945, cuando los Estados Unidos obtuvieron el arma nuclear. Fue a partir de la carrera de armamento nuclear entre la Unión Soviética y los Estados Unidos cuando la posesión de amplios arsenales estratégicos otorgó a estos Estados la condición de "superpotencias" y abrió una profunda brecha entre éstas y el resto de potencias con responsabilidades mundiales ${ }^{37}$ Sin embargo, la "estrategia de la disuasión" que dominó las relaciones Este-Oeste durante cuarenta y cinco años (1945-1990) estaba basada en la asunción de que era la posesión, y no el uso del arsenal estratégico, lo que resultaba suficiente para detener el ataque ${ }^{38}$. Por tanto, de lo que no hay duda es de que el poderío militar -también denominado "poderío beligerante" ${ }^{39}$ - de un país parece ser la prueba final de su status en la jerarquía de los Estados ${ }^{40}$. Con todo, debemos afirmar que el poderío militar

\footnotetext{
${ }^{34}$ Cit. en Garnett: "The role of military power", op. cit., p. 73; en idéntico sentido, Betts: "La nueva amenaza de destrucción masiva", Pol. Ext. núm. 63, 1998, pp. 135-151, en concreto, p. 131.

3s Vid. al respecto el excelente artículo de Remiro Brotons: "Civilizados, bárbaros y salvajes en el nuevo orden mundial", op. cit.

${ }^{36}$ Howard: "Military Power and International Order", Int. Aff. nüm. 3, 1964, p. 405.

37 Vid, en este punto, todo en general, Nadal Egea: Arsenales nucleares. Tecnología decadente y control de armamentos. El Colegio de México. México, 1991; también Gallois: Geopolítica, op. cit., pp. 365-386. Para el inicio de esta carrera Thomas: Paz armada. Los comienzos de la guerra fría (1945-i946). Ed. Grijalbo. Barcelona, 1988 (trad. de Armed Truce. Hamish Hamilton. Londres, 1986), pp. 451-496.
} 
de un Estado no es ni bueno ni malo, éticamente es neutral. Es bueno o malo en la medida que se use para buenos o malos propósitos.

Pero no sólo el poderío militar da a un Estado su condición de potencia. Para acceder a esta condición una Nación debe contar con excelentes recursos materiales, industriales y militares, sustentados en una base económica floreciente. ${ }^{41}$ Efectivamente, un principio fundamental de las relaciones internacionales establece que cuanto menores sean las capacidades económicas de un Estado, mayores serán sus dificultades para mantener opciones políticas propias y ejercer su independencia en el sistema internacional. A principios de este siglo Amery advirtió que "las potencias triunfales serán aque1las que tengan la mayor base industrial. Los pueblos que tengan la fuerza

${ }^{38}$ En teoría de los juegos, una suerte de juego de la gallina frustrado. Sobre esta cuestión, el mariscal Grechko escribía en los años setenta: "El poderío combativo [de las fuerzas armadas soviéticas] se basa en las Tropas Coheteriles Estratégicas que con la mayor plenitud han asimilado los últimos adelantos del progreso científico y técnico". (Las fuerzas armadas del Estado soviētico. Ed. Progreso. Moscú, 1977, pp. 84-85). Por su parte en 1981 el Secretario de Estado americano Brown se expresaba en los siguientes términos: "nuestra capacidad nuclear estratégica es la base sobre la que se asienta nuestra seguridad [...]. Con ella, nuestras otras fuerzas pasan a ser instrumentos importantes del poder político y militar" (cit. en Chomsky et all.: Superpotencias en colisiōn. La nueva guerra fría en los años ochenta. Ed. Debate. Madrid, 1985 (trad. de Superpowers in collision. Penguin Books, 1984) p. 75). Sobre la cuestión del "uso" de armas nucleares en España, vid. García Rico: La cuestión del primer uso de las armas nucleares en el Derecho internacional (tesis doctoral inédita). Sevilla, 1995; y la obra colectiva La licitud del uso de las armas nucleares en los conflictos armados. IV Jornadas de Derecho Internacional Humanitario (Fernández Sänchez coord.). Univ. de Huelva-Univ. de Sevilla-Cruz Roja Española-Ministerio de Defensa. Sevilla, 1997. Finalmente, por la actualidad de la cuestión que plantea, Slocombe: "¿Es aún necesaria la disuasión nuclear?", Revista de la OTAN núm. 6, 1997, pp. 23-26.

39 Segün una declaraciōn de cinco expertos britänicos en el ámbito de la organizaciôn militar formulada en 1943, el "poder beligerante" de una nación se define como "la suma de su poderío maritimo, terrestre y aéreo empleado colectivamente y en verdadera cooperación, más su poder civil. Todos los elementos del poder beligerante, la Armada, el Ejército, la Fuerza Aérea, la Marina Mercante, la Defensa Civil, el comercio, las finanzas, la industria, la propaganda, las materias primas, los productos alimenticios, las comunicaciones, los científicos, los proyectistas e inventores y, en verdad, todos los que trabajan para la guerra están interrelacionados y dependen unos de otros así como la dirección. El poder beligerante se ejerce mediante la organización, el entrenamiento, el equilibrio, la coordinacioon y la dirección de todas las manifestaciones del esfuerzo nacional, para lograr los máximos resultados en la realización de un fin único", podríamos añadir la victoria, o mejor, la supervivencia de la Nación (cit. en Schwarzenberger: La política del poder, op. cit., p. 104).

40 Fox: Las superpotencias, op. cit., p. 15; Morgenthau: La lucha por el poder y por la paz, op. cit., p. 163; Schwarzenberger: La política del poder, op. cit., p. 104; Spykman: Estados Unidos frente al mundo, op. cit., pp. 25.26 y 29; Vital: La supervivencia de los pequeños Estados. Instituto de Estudios Políticos. Madrid, 1975 (trad. de The survival of small States. Oxford University Press. Londres, 1971), p. 17. De todas formas, el mismo Morgenthau advirtió de los peligros de considerar la potencia militar como factor único del poder de la nación (La lucha por el poder y por lá paz, op. cit., pp. 221-224).

${ }^{41}$ Sobre esta idea gira la obra de Kenriedy, Auge y caída de las grandes potencias: "ciertamente, el resultado de todas las grandes y prolongadas guerras entre grandes potencias que se han estudiado aquî indica repetidamente las influencias cruciales de las fuerzas económicas productivas, tanto durante la propia contienda como durante aquellos períodos de entre guerras, cuando los índices diferenciados de crecimiento hacen que las diversas potencias se fortalezcan o debiliten relativamente". (op. cit., p. 833, la cursiva es nuestra). 
industrial y la fuerza de invención y de ciencia serán capaces de derrotar a todos los demás." 42 Si a lo largo de la Historia este hecho había sido una premisa fundamental a la hora de valorar el poderío de los Estados a largo plazo, es en el siglo XX cuando la productividad industrial, junto con el desarrollo de las ciencias experimentales y la tecnología, se convierte en elemento decisivo en la configuración de la fuerza de los Estados. ${ }^{43} \mathrm{La}$ mejor prueba de esta afirmación la tenemos en la Unión Soviética tras el final de la guerra civil con la puesta en marcha de los dos Planes Quinquenales (1928-1937). En efecto, los extraordinarios resultados de estos Planes transformaron a la Unión Soviética "de una nación agraria a una nación industrializada", como puso de manifiesto Stalin en enero de 1934 durante la celebración del XVII Congreso del PCUS. Por muy cuestionables que resulten las cifras alcanzadas, no cabe duda de que había surgido una nueva gran potencia industrial y, como acertadamente expresa Nolte, "por esta razón estaba encaminada a erigirse en una potencia política y militar a nivel mundial". Sin este desarrollo industrial la Unión Soviética no hubiera podido acceder al rango al que aspiraba en el sistema internacional, al menos desde la época de Pedro el Grande. ${ }^{44}$

De todas formas, en los años setenta se pretendió acuñar la noción de "potencia civil" 45, desarrollada especialmente en la década siguiente, cuando dentro del sistema bipolar de las relaciones internacionales se produjeron una serie de "movimientos antilíder" ${ }^{46}$, particularmente en el bloque occidental. Con este término se hacía referencia a aquellos Estados con grandes recursos materiales, industriales, financieros y sociales, pero carentes de una fuerza militar proporcionada a su tamaño. De esta manera, se quería desvincular la

${ }^{42}$ Cit. en ibid, p. 319.

${ }^{43}$ Como dijo List "la guerra o la mera posibilidad de guerra hace que el establecimiento de un poder fabril sea requisito indispensable para una nación de primera categoria" (cit. en ibid,., p. 837). Como dato ilustrativo, las empresas privadas americanas, ayudadas por los fondos del gobiemo federal, consiguieron que el PNB de los Estados Unidos aumentara más del doble en el período de $1941 \cdot 1945$.

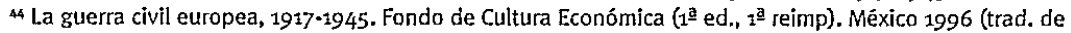
Der europäische Bürgerkrieg 1917-1945. Nationalsozialismus und Bolschewismus. Verlag Ullstein. Francfort del Meno, 1987), p. 206; en este sentido, vid. también Grechko: Las Fuerzas Armadas del Estado Soviétjco, op. cit., pp. 41.53, esp. p. 46 y 47; Kennedy: Auge y caída de las grandes potencias, op. cit., pp. 510 . 515; Morgenthau: La lucha por el poder y por la paz, op. cit., p. 161.

45 El primero en utilizar este término fue Duchène: "Europe"s Role in World Peace", Europe Tomorrow: Sixteen Europeans Looks Ahead (Mayne comp.). Fontana, Londres, 1972, pp. $42-47$.

46 Barbé: Relaciones Internacionales, op. cit, p. 243.

47 lbid, pág. 145; Garnett: "The role of military power", op. cit., p. 76. Efectivamente, "potencial militar" no es lo mismo que "fuerza militar". Una gran potencia económica puede optar por permanecer como un "enano" militar, mientras que un Estado con pocos recursos económico-productivos puede organizar una formidable potencia militar (Kennedy: Auge y caïda de las grandes potencias, op. cit., p. 321). 
noción de potencia-como hemos dicho anteriormente, ejercicio de influencia y autoridad en el sistema- de la capacidad militar ${ }^{47}$. Para los autores que han analizado la cuestión, la noción de "potencia civil" -o "Estado comercial", como también se ha denominado ${ }^{48}$ - implica: 1) el reconocimiento de la necesidad de una cooperación amplia con otros actores del sistema internacional; 2) la concentración en los asuntos no militares, fundamentalmente los económicos, de la seguridad nacional, estableciendo una fuerza militar de carácter residual para salvaguardar los intereses esenciales de la seguridad ${ }^{49}$; y 3) la capacidad de cooperación avanzada en las estructuras internacionales donde resolver las cuestiones críticas del sistema internacional ${ }^{50}$. Sin embargo, esta noción es, a nuestro juicio, una falacia por cuanto el status de potencia lo obtiene un Estado cuando escala por encima de sus iguales en todos -o en casi todos-los indicadores económicos, industriales e, indudablemente, militares ${ }^{51}$, pues incluso aquellos Estados que podrían ser calificados como potencias civiles tienen unas fuerzas armadas y unos recursos militares muy superiores a los de sus vecinos. ${ }^{52}$ Efectivamente, en la actualidad podemos com-

\footnotetext{
${ }^{48}$ Término acuñado por Rosecrance: La expansión del Estado comercial. Comercio y conquista en el mundo moderno. Alianza Ed. Madrid, 1987 (trad. de The rise of the trading State. Commerce and conquest in the modern world. Basic Books. Nueva York, 1986).

49 Como afirma Rosecrance: "los Estados que se apuntan a la estrategia comercial, también tienen que cubrir su flanco defensivo, pero en lugar de actuar aisladamente, recurren al mismo modelo de cooperación, estableciendo alianzas para prevenir una posible agresión de las naciones político-militares" (ibid., p. 39). ${ }^{50} \mathrm{Vid}$. al respecto Maull: "Germany and Japan: the new civilian powers", For. Aff. núm. 5, 1990, pp. 91-106. Para este autor Alemania y Japón se han convertido en los prototipos de un "promisorio" nuevo orden internacional; es más, declara fenecido el concepto/rôle internacional de superpotencia (sic) (ibid., p. 92); suponemos que ahora utilizará el término "potencia hegemónica" o, mejor, "potencia global" ...

${ }^{51}$ Los defensores de la noción de "Estado comercial" aseguran que la guerra no compensa los sacrificios realizados porque los intercambios comerciales constituyen el mejor medio para aumentar la riqueza y el poderio de las naciones. Sin embargo, como afirman layne y Schwarz, la comunidad de la política exterior de los Estados Unidos ha dado la vuelta a este esquema. Así, la estrategia americana del orden mundial del final del siglo "está basada en la creencia de que EE.UU. debe mantener lo que es esencialmente un protectorado militar en regiones de gran importancia económica, para garantizar que las relaciones financieras y comerciales para EE.UU. no se vean afectadas por disturbios políticos. [...] Los expertos en política exterior consideran que el poder militar de EE.UU. debe imponer la armonía para que pueda producirse el libre comercio." ("Sin enemigos: la nueva hegemonía norteamericana", Pol. Ext. núm. 37. 1994, pp. 83-99, en concreto, pp. 89-90).

\$2 Hablamos en concreto de Alemania y Japón, considerados ambos potencias regionales y dotados de unas fuerzas armadas y unas capacidades de movilización en caso de conflicto muy superiores a las de los Estados de su entorno; Brzezinski llega a calificarlos de "cuasipoderes globales" (El gran tablero mundial, op. cit., pp. 179-180). Vid. al respecto los datos estadísticos en Balance Militar 1995.96. Ministerio de Defensa. Madrid, 1996 (trad. de Military Balance. The International Institute for Strategic Studies. Londres, 1995), pp. $55-58$ y $236 \cdot 239$. Sobre Japón, vid. el interesante artículo de Hamada: "La Constitución pacifista del Japón de posguerra idividendos económicos o cargas políticas?", Desarme núm. 3, 1996, pp. 42-57. Con respecto al papel de China como "presunta" potencia militar mundial, vid. Brzezinski: "Una estrategia para Eurasia", Pol. Ext. núm. 60, 1997, pp. 157-173, esp. pp. 168-172.
} 
probar como la Federación Rusa goza del status de gran potencia sobre todo gracias al inmenso arsenal nuclear heredado de la guerra fría -según los servicios de inteligencia franceses y americanos, Rusia dispondría aún de 6.640 cabezas nucleares estratégicas y 20.000 tácticas-. Su influencia internacional sería, sin lugar a dudas, muy inferior si careciese de fuerzas nucleares estratégicas y de su aparato militar-industrial. ${ }^{53}$ Por tanto, como ha expresado Kennedy ${ }^{54}:$ "[...] el triunfo de cualquier gran potencia [...], o el colapso de otra ha sido la consecuencia de prolongadas luchas de sus fuerzas armadas, pero también la utilización más o menos eficiente de los recursos económicos productivos del Estado en tiempos de guerra y, más en segundo término, la consecuencia de la forma en que la economía de ese Estado había estado mejorando o empeorando en relación con las de otras naciones líderes durante las décadas que precedieron al conflicto armado."

Así pues, continúa este autor, "hay una relación causal detectable entre los cambios que se han producido en el tiempo en los equilibrios económicos y productivos generales y la posición ocupada por las potencias individuales en el sistema. [...] De la misma manera [...] a largo plazo hay una conexión muy evidente entre el ascenso y caída económicos de una gran potencia y su crecimiento y declive como poder militar importante, o imperio mundial." 55 En definitiva, en el momento actual de desarrollo del sistema internacional un cambio en el rango del nivel de desarrollo económico general, hacia arriba o hacia abajo en la escala, irá acompañado por su correspondiente cambio en la jerarquía de poder en la sociedad internacional.

53 En este sentido, Kissinger: Diplomacia, op. cit., pp. 836; vid. datos al respecto en Balance Militar 199596, op. cit., pp. 137-138 y 146-155; tambiên, Norris y Arkin: "Global Nuclear Stockpiles, 1945-1997", The Bulletin of Atomic Scientists, nov./dic. 1997, p. 67. Sobre la reforma de las FAS de la Federación de Rusia tras el final de la Guerra Fría, vid. Sergeyev: "Socios, no adversarios", Revista de la OTAN núm. 1, 1998, pp. 15-18. Como expresa Betts "ahora las cosas han camblado. Ya no hay Pacto de Varsovia, Rusia tiene una capacidad militar menor que la que tenía la Unión Soviêtica y sus fuerzas convencionales están sumidas en el caos [...] Es Moscú ahora (paradójicamente] quien tiene que basarse más en la capacidad nuclear". (en "L.a nueva amenaza de destrucciốn masiva", op. cit., p. 142). Sobre el papel de la Federación Rusa en el sistema de seguridad emergente, ya expresamos nuestras consideraciones en otro lugar: "Hacia un nuevo sistema de seguridad global", Anales de la Facultad de Derecho de la Universidad de La Laguna núm. 14, 1997, pp. 219-240, esp. pp. 226-227.

${ }^{54}$ Kennedy: Auge y caída de las grandes potencias, op, cit., pp. 9:10.

s5 bid,, pág. 20; Remiro Brotons: "Civilizados, bărbaros y salvajes en el nuevo orden internacional", op. cit., p. 70 . 


\section{La voluntad política para ejercer como potencia}

Más arriba expresamos que son las potencias quienes efectivamente disponen del poder estructural para dictar las reglas del sistema internacional. Pero si el poder es un requisito fundamental, el elemento esencial que le otorga a un Estado la condición de potencia es la voluntad de usar dicho poder ${ }^{56}$, para nosotros el elemento subjetivo. Como ha dicho Holbraad, "si el poder de una nación se define como su capacidad de imponer su voluntad sobre otros Estados, y de resistirse a los intentos de otros Estados por imponerse sobre ella, la fuerza de una nación puede definirse como los medios con los cuales ejerce su poder" 57 , y el elemento indispensable para ser una potencia, "el elemento operativo, es la voluntad y la capacidad para usar el poder imponiendo un cierto orden en la escena internacional" 58 y además, estar dispuesto a hacer frente a su costo. ${ }^{59}$ Se trata sin duda del más evasivo de los factores que conforman el poder nacional, pero sin su concurso ningún gobierno, democrático o autoritario, puede lograr de forma efectiva sus metas políticas. Por tanto, para que un Estado escale al status de potencia constituye un elemento fundamental, decisivo a nuestro juicio, que sus elites dirigentes y población crean que su sociedad es un modelo para las demás. ${ }^{60}$ Ya Tucídides destacó la importancia de este factor en su Oración Fúnebre de Pericles, pronunciada en el entierro de los atenienses muertos en la primera guerra del Peloponeso: "tenemos un régimen político que no emula las leyes de otros pueblos, y más que imitadores de los demás, somoś un modelo a seguir [...] Resumiendo, afirmo que nuestra ciudad es, en su conjunto, ejemplo para Grecia." ${ }^{61}$ En este

\footnotetext{
${ }^{56}$ Merle: Sociología de las relaciones internacionales, op. cit., p. 351.

57 Holbraad: Las potencias medias en la política internacional, op. cit., p. 95; en el mismo sentido, Deutsch: Análisis de las relaciones internacionales, op. cit., p. 41-42; Vital: La desigualdad de los Estados, op. cit., p. 137. $5^{8}$ Pfaff: "The Might and Sense of a Superpower", The International Herald Tribune, 14 diciembre 1990. Pfaff hacia referencia a la imposibilidad de los Estados Unidos para hacer frente, ellos solos, al costo del despliegue de una fuerza militar destinada a expulsar a Irak de Kuwait. Sobre el "reparto de cargas" entre la potencia hegemónica y sus adláteres, vid. el breve pero contundente artículo de Sloan: "Puede enturbiar las relaciones transatlânticas el proceso de ampliación?", Revista de la OTAN núm. 5, 1997, pp. 12•16.

s9 Schwarzenberger: La política del poder, op. cit., p. 205.

6o Kennedy habla también de la cohesión nacional, el patriotismo y el control ejercido por el Estado sobre sus habitantes, pero estos elementos se engloban en lo que hemos denominado "la creencia de una sociedad de que es un modelo para las demás" (Auge y caída de las grandes potencias, op. cit., p. 320); también Brzezinski: El gran tablero mundial, op. cit., pp. 19-38.

${ }^{61}$ Historia de la guerra del Peloponeso. Ed. Gredos. Madrid, 3990, pp. 450 y 455. Sun Tze en El arte de la guerra expresó la misma idea: "lo que más contribuye a la fortaleza de un país es tener sus propias ideas, su propia visión del mundo; y despuës encontrar alianzas que le den estabilidad y apoyo moral para sostener sus esfuerzos".
} 
siglo, esta idea fue puesta magistralmente de manifiesto por Kennan refiriéndose a su propio país, en el momento en que los Estados Unidos tuvieron que hacer frente a las responsabilidades como gran potencia tras el final de la Segunda Guerra Mundial (1947) ${ }^{62}$ :"(...) en realidad las posibilidades de la política estadounidense no se limitan de ningún modo a mantener una posición y esperar que todo salga bien. (...) Se trata de la capacidad de los Estados Unidos para proyectar en los pueblos del mundo la imagen de un país que sabe lo que quiere, que se enfienta victoriosamente a sus problemas internos y a las responsabilidades de una potencia mundial, y que tiene una vitalidad espiritual capaz de estar a la altura de las más importantes corrientes ideológicas de nuestro tiempo."

El fin de la guerra fría ha reafirmado en la comunidad de la política exterior esta convicción del embajador americano. En el documento National Security Strategy of the United States de agosto de 1991, el presidente Bush afirmaba: "Tenemos al alcance de nuestra mano una extraordinaria posibilidad que pocas generaciones han disfrutado: edificar un nuevo sistema internacional conforme con nuestros propios valores e ideales"; reiterando en la National Security Strategy de 1993 que "Ninguna otra Nación tiene la misma capacidad de credibilidad moral, cultural, política, económica y militar. Ninguna otra ha alcanzado semejante confianza, respeto y fidelidad. Ninguna otra tiene el mismo potencial y la responsabilidad para ejercer el liderazgo mundial." 63 Estas consideraciones son extrapolables a cualquier Estado que aspire a ejercer algún grado de hegemonía internacional. ${ }^{64}$ También en España, Aldecoa ha puesto de manifiesto esta idea cuando destaca, como uno de los elementos fundamentales en el análisis del concepto de potencia, "la voluntad política de desempeñar un papel activo en la sociedad internacional." 65

\footnotetext{
${ }^{62}$ Kennan: "The Sources of Soviet Conduct", For. Aff. núm. 4, 1947, pp. 566-582. Este artículo supuso, ante todo, una reacción contra la concepción idealista de la política exterior de los Estados Unidos mantenida con ciertas oscilaciones hasta el final de la Segunda Guerra Mundial. En este sentido, vid. reciente Layne y Schwarz: "Sin enemigos: la nueva hegemonia norteamericana", op. cit., pp. 83-99.

${ }^{63}$ The White House. Washington, 1991, p. v, y 1993, p. 21. Como no podía ser de otra manera, el último documento de seguridad nacional de presidente Clinton se emplea en idénticos términos (vid. las conclusiones de A National Security Strategy for a New Century, op. cit.).

${ }^{64}$ En este sentido expresa Kissinger que "como los norteamericanos, también los rusos consideraban que su sociedad era excepcional" y, por tanto, exportable al resto de naciones del planeta (Diplomacia, op. cit., p. 144). Con carácter general, Deutsch: Análisis de las relaciones internacionales, op. cit., pp. 132. 133 ; Morgenthau: La lucha por el poder y por la paz, op. cit., pp. 182-190; Spykman: Estados Unidos frente al mundo, op. cit., p. 26.
} 
Por el contrario, el agotamiento no sólo material sino particularmente moral y psicológico de las elites dirigentes del Estado acarrea el descenso en la jerarquía internacional de las grandes potencias, abocando al Estado, en un proceso más o menos rápido, a la condición de potencia de segundo orden y, finalmente, a mero sujeto carente de capacidad de influencia en el sistema internacional tal como entendemos ésta hasta ahora. Con la lección aprendida, el presidente Bush consideraba en la National Security Strategy de 1993 que para que los Estados Unidos continúen en el siglo que viene ejerciendo el liderazgo indiscutido en el sistema internacional será menester "renovar nuestra vitalidad interior -en productividad económica, tecnología, educación y energía- como un prerrequisito absoluto de nuestro futuro." 66

Para concluir, podemos decir que el poder de un Estado en modo alguno reside exclusivamente en el volumen de sus fuerzas armadas, sino también en sus recursos económicos, financieros y tecnológicos; en la destreza, la previsión y la resolución con que se dirige su política exterior, y en la eficacia de su organización política y social. Consiste sobre todo en la propia nación, en el pueblo; en su habilidad, su energía, su ambición, su disciplina, su iniciativa; sus creencias, mitos e ilusiones. Y consiste además en la manera en que todos estos factores se combinan entre sí. ${ }^{67}$

\footnotetext{
6s Aldecoa: "La construcción de la política exterior española", Política y Sociedad 2, 1989, p. 68.

66 op. cit., p. ii

${ }^{67}$ Barnett: The Collapse of British Power. Londres, 1972, p. XI.
} 


\section{La jerarquía entre las potencias}

Como se ha puesto de manifiesto, el tema tratado es de una indudable complejidad. Por este motivo, todos los autores han tendido a adjetivar el fenómeno de las potencias ${ }^{68}$, circunstancia que implica, evidentemente, una estimación comparativa del poder del Estado basada en un inventario de los elementos analizados más arriba; se habla entonces de "potencias hegemónicas," 69 " superpotencias", " grandes potencias", " potencias medias", " potencias regionales" $\mathrm{y}$, contradictoriamente, "pequeñas potencias" 70 .

Aunque politólogos e internacionalistas han desarrollado diversos métodos para medir el peso de los Estados en la sociedad internacional y han procedido a clasificarlos en un orden jerárquico, el Derecho Internacional no reconoce estas divisiones. Es cierto, también, que la valoración del poderío estatal no se puede confiar a un solo factor del poder estatal. Como hemos expresado hasta ahora, para acceder al concepto de potencia debemos tener en cuenta los factores geográficos y demográficos, militares y económicos y, finalmente, los políticos y de recursos humanos. Los primeros pueden ser cuantificables y, por tanto, son considerados más o menos objetivos. Por el contrario, los factores políticos y de cohesión del grupo humano que forma el elemento subjetivo del Estado son intangibles y su valoración adolece inevitablemente de un alto grado de subjetividad y arbitrariedad lo que dificulta el mejor de los análisis. Al mismo tiempo, algunos factores son relativamente estables, y empleamos el término "relativamente" por cuanto nos encontramos ante un fenómeno que debe ser fijado en el tiempo para poder ser analizado con garantías de éxito; otros factores se encuentran sujetos a variaciones en plazos de tiempo más cortos..$^{71}$

La valoración y cuantificación de los factores geográficos y de recursos naturales no plantea, en principio, demasiados problemas debido a su estabi-

\footnotetext{
68 Barbé: Relaciones Internacionales, op. cit., p. 147.

6 Brzezinski emplea el término "potencia global" para referirse al masivo poder imperial de que hacen gala los Estados Unidos en el sistema internacional global de finales del siglo XX (todo en general, El gran tablero mundial, op. cit.).

70 Para Vital aquellos Estados "que carecen de recursos y capacidad para representar un papel internacional independiente, son verdaderas pequeñas potencias, y las que no lo hacen no son pseudo pequeñas potencias, [...." El autor reconoce que se trata de "una definición francamente subjetiva, si no arbitraria. Pero es la que está apoyada por el sentido común [sic]". (La desigualdad de los Estados, op. cit., pp. 24-25). n Vid. al respecto Deutsch: Análisis de las relaçiones internacionales, op. cit., pp. 62-64; Morgenthau: La lucha por el poder y por la paz, op. cit., pp. 2:0-216.
} 
lidad en el tiempo; sólo profundos cambios tecnológicos inciden en su estabilidad como atributos permanentes del poderío del Estado. ${ }^{72}$ Para los factores económicos y de recursos materiales, el indicador más fiable y recurrentemente utilizado es el del Producto Nacional Bruto (PNB). ${ }^{73}$ Esta cantidad refleja la mayor parte de los factores materiales que componen el poder de un Estado en esencia es una medida de fuerza económica- $y$, aunque en la actualidad el índice del PNB tiende a ser complementado por el Indice de Desarrollo $\mathrm{Hu}$ mano (IDH), su fiabilidad lo mantiene como criterio de clasificación prioritario $^{74}$. Otras ventajas adicionales son que se trata de una cantidad cuidadosamente calculada -en la mayoría de los casos es así- y de la que se dispone con gran facilidad. Con todo, el PNB no refleja adecuadamente todos los puntos fuertes y débiles del poder de un Estado. Así a corto plazo el volumen de las Fuerzas Armadas, el gasto militar y la cantidad de armamentos indican el poder militar real del momento. A largo plazo, el tamaño de la población puede ser un indicador más fiable, al complementarlo con el volumen del PNB.$^{75}$ Resulta claro, por tanto, que no existe un único indicador fijo y exclusivo por el que se pueda determinar de forma matemática qué Estados se sitúan en el status de potencia ni el lugar que ocupan en la jerarquía ${ }^{76}$. Al final la evaluación del poder continúa siendo relativa y dependerá siempre de un juicio puramente subjetivo. De esta forma, como afirma Barnett, "el poder nacional tiene que ser considerado no únicamente en sí mismo, en su extensión abso-

\footnotetext{
72 Para Spykman "la geografía es el factor fundamental para la política exterior de los Estados porque es el más permanente". (Estados Unidos frente al mundo, op. cit., p. 47); en idéntico sentido Morgenthau: La lucha por el poder y la paz, op. cit., p. 151. Sin embargo, Bowman expresó que "a menudo se dice que la geografía [la base fisica del Estado] no cambia. Pero la verdad es que la geografía cambia al ritmo de las ideas y [sobre todo] de la tecnología; lo que quiere decir que el significado de las condiciones geográficas cambia". ("The geographical situation of the United States in relation to World Politics", Geog. Jour. nüm. 112, 1948, pp. 129-142, en concreto p. 130).

73 En este sentido p.e., Barbé: Relaciones Internacionales, op. cit., p. 135; Deutsch: Análisis de las relaciones internacionales, op. cit., p. 45.

${ }^{74}$ El IDH apareció en 1990 a raíz de una iniciativa del PNUD y el gobiemo sueco. La tarea encargada al economista pakistani Mahbud ul-Haq consistía en crear un nuevo índice para juzgar, país por pais, lo que el crecimiento económico hace en favor de los pueblos centrándose en tres indicadores principales: esperanza de vida, alfabetizaciôn y renta real -calculada por el poder adquisitivo- (vid. al respecto, Cleveland: Nacimiento de un nuevo mundo. El Pais-Aguilar. Madrid, 1994 (trad. de Bith a new World. Jossey-Bass Inc., 1993), pp. 222-225).

75 Holbraad: Las potencias medias en la política internacional, op. cit., pp. 97.99. Para Morgenthau, "ningún país puede llegar a ser una potencia de primer orden si no se cuenta entre las más pobladas del mundo" (La lucha por el poder y por la paz, op. cit., pp. 168-170). En cualquier caso, es preciso hacer notar que el volumen de la poblaciôn determina ciertos límites a la acciôn exterior de los Estados y fija el potencial económico de los mismos. En este sentido, vid. Reynolds: Introducción al estudio de las relaciones internacionales, op. cit., pp. 82-90.
} 
luta, sino relativa a las obligaciones exteriores imperiales del Estado; tiene que ser considerado en relación con el poder de otros Estados." 77 Por este motivo resulta obvio que los diversos Estados no pueden ser, de forma simultánea, más poderosos: lo que gana uno necesariamente lo pierde otro. ${ }^{78}$ En consecuencia, la expansión del nivel de poder de los Estados, especialmente a partir de 1945, ha hecho que toda política exterior sea mucho más costosa. Si para establecer el orden jerárquico de los Estados el primer paso consiste en clasificarlos conforme a un indicador -el que sea científicamente aceptable-, el siguiente en trazar líneas divisorias en la jerarquía de poder. La línea superior es clara y en pocas ocasiones plantea discusión, pues se encuentra formada por las grandes potencias del sistema -llámense como se llamenque no dudan en hacer sentir a los demás sujetos de la sociedad internacional su "presencia". Su posición, reconocida hoy en día por el Derecho Internacional ${ }^{79}$ y respetada por la práctica diplomática, se caracteriza por una sólida conciencia de clase y, a pesar del enfrentamiento a que se suelen ver aboca-

${ }^{76}$ Wood realizó una clasificación de las potencias medias basada exclusivamente en el PNB (en "Towards North-South Middle power coalition", Middle power internationalism: The North-South dimension (Pratt ed.). McGill-Queen University Press. Montreal, 1990). Asī, estableció como potencias medias a treinta Estados que se encontraban inmediatamente después de las grandes potencias (el autor determinó seis de éstas). De esta manera, como puso de manifiesto Palou, incluyó en la lista a Estados, especialmente europeos, que ningún otro autor considera como potencias medias, pero dejó de lado p.e. a Egipto (en "El concepto de potencia media: España y México.", Af. Int. núm. 26, 1993, pp. 7-35, en concreto pp. 1718). Evidentemente prescindir de otros indicadores, que deben ser tenidos en cuenta conjuntamente a efectos de determinar la jerarquía de las potencias, lleva a resultados poco satisfactorios.

7 The Collapse of British Power, op. cit., p. Xl; también Morgenthau: La lucha por el poder y por la paz, op. cit., pp. 204-223; Reynolds; Introducción al estudio de las relaciones internacionales, op. cit., pp. 125-131; Schwarzenberger: La política del poder, op. cit., p. 105; Spykman: Estados Unidos frente al mundo, op. cit., p. 29. ${ }^{78}$ En este sentido, Fox: Las superpotencias, op. cit., pp. 22-24; y todo en general Kennedy: Auge y caîda de las grandes potencias, op. cit.

29 En contra, opinaba Oppenheim que "ningún Estado tiene carácter de gran Potencia en virtud del Derecho. Son solamente la extensión, la fuerza y la influencia económica las que hacen de un Estado una gran Potencia". Sin embargo, el mismo autor ponía de manifiesto que tanto el Pacto de la SdN como la Carta de la ONU confirmaron jurídicamente el status que de facto gozaban las grandes potencias del momento: "[...] aparte del Pacto de la Sociedad de las Naciones, la hegemonía de las grandes Potencias era, antes de la Carta de las Naciones Unidas, de carácter político pero no jurídico". (Tratado de Derecho Internacional Público, op. cit., pp. 293-294). En idéntico sentido, Russett y Starr: World Politics. The Menu for Choice, op. cit., pp. 60-64. En España, Pastor Ridruejo constata que "por lo que respecta a la composiciōn del Consejo [de Seguridad] encontramos en la Carta una consagración formal de la desigualdad real entre Estados". (Curso de Derecho Internacional Público y Organizaciones Internacionales, op. cit., p. 686); como afirma Remiro Brotons: "cinco Estados, los miembros permanentes del Consejo de Seguridad, estân por encima de las leyes internacionales, con un estatuto privilegiado que los hace dueños de la llave del poder constituyente, como también lo son de toda letalidad nuclear oficialmente reconocida y del 80 por 100 del comercio de armas convencionales. Sin embargo, ni siquiera así la Organización es concebida por ellos como la clave de bóveda del sistema internacional de paz y seguridad, sino sólo como uno de los instrumentos complementarios de su política" ("Civilizados, bárbaros y salvajes en el nuevo orden internacional.", op. cit., pág. 79). 
das, la comunidad de intereses que mantienen.$^{80} \mathrm{El}$ número de estas potencias en la sociedad internacional ha sido y es, obviamente, muy limitado. Por el contrario, las potencias medias -locally predominant powers en la terminología anglosajona- no gozan del reconocimiento jurídico o convencional del que hacen gala las grandes potencias y, por tanto, trazar la línea inferior de separación entre éstas y el resto de los Estados no resulta una cuestión pacífica. La solución pasa inevitablemente por la consideración de las potencias medias en una escala regional ${ }^{81}$. Fuera de este marco hablamos simplemente de Estados, Estados pequeños o, incluso, "microestados". 82

\footnotetext{
${ }^{80}$ Vid. al respecto las interesantes líneas de Fox: Las superpotencias, op. cit., pp. 189-208; Schwarzenberger: La política del poder, op. cit., pp. 99-108. En esta cuestión, Hambro, presidente del Parlamente noruego, expresaba en 1944: "toda pequeña nación tiene que abandonar la idea tan acariciada de que su influencia en los asuntos internacionales debe ser tan grande como la de cualquier otra nación [...] En cualquier organizaciôn universal, independientemente de su nombre, pocos son los grandes países que llevan sobre los hombros la responsabilidad de hacer cumplir las decisiones de la autoridad mundial, y es a estos paises a los que debe otorgarse, constitucionalmente, el poder formal correspondiente a su responsabilidad real". (cit. en Fox: Las superpotencias, op. cit., pp. 192-193).

a Vid. la problemática de esta cuestión in extenso en Holbraad: Las potencias medias en la política internacional, op. cit., pp. 99-113; y todo en general Vital: La desigualdad de los Estados, op. cit.

${ }^{B_{2}}$ No obstante, de forma paradójica, con todo el potencial de destrucción acumulado por las dos superpotencias durante la guerra fria, los Estados más pequeños han podido continuar con sus disputas y guerras regionales con relativa impunidad, por cuanto por su propia naturaleza no seria politicamente factible la amenaza de uso o el uso de los arsenales de destrucción masiva contra estos Estados recalcitrantes. Harden ha puesto de manifiesto que las grandes potencias subestiman grandemente los problemas que suscitan los microestados y esta situación puede ser origen, y de hecho lo es, de "macrodesórdenes" en la organización internacional -especialmente, en las Naciones Unidas- y en las grandes capitales (Small is Dangerous. Micro States in a Macro World. Frances Pinter, Londres, 1983, p. 4). Además, Vital llega a hablar de "micropotencias", lo cual no deja de ser como minimo un contrasentido, citando como ejemplo a Samoa Occidental (sic) (La desigualdad de los Estados, op. cit., pp, 26-27). Finalmente, para el Derecho internacional los Estados "exiguos" constituyen otra categoria con problemas propios, distintos y caracteristicos, pero fuera de nuestro objeto de estudio.
} 